PALABRAS CLAVE

Tasas de interés

Préstamos bancarios

Bancos comerciales

Análisis económico

Política monetaria

Bancos centrales

Bahamas

Barbados

Nlandu Mamingi

Profesor de Economía, Departamento de Economía

Universidad de las Indias Occidentales,

Bridgetown, Barbados

œ Nlandu.mamingi@cavehill.uwi.edu

Daniel O. Boamah

Vicepresidente del Banco Central de

Barbados

•DOBoamah@centralbank.org.bb

Mahalia N. Jackman

Economista del Departamento de

Investigaciones

del Banco Central de Barbados

œMNjackman@centralbank.org.bb
REVISTA CEPAL 103 • ABRIL 2011

\section{Bahamas y Barbados: evidencia empírica de la transmisión de las tasas de interés}

\author{
Nlandu Mamingi, Daniel O. Boamah y Mahalia N. Jackman
}

A

fin de investigar empíricamente la eficacia de la política de tasas de interés de los bancos centrales de las Bahamas y Barbados para influir en las tasas sobre los préstamos de los bancos comerciales, se analizaron los datos trimestrales entre enero de 1995 y abril de 2007 mediante un modelo de corrección de errores. En Barbados las tasas de los préstamos de los bancos comerciales no responden a corto plazo ante los cambios de la tasa de interés de política monetaria del banco central, pero a largo plazo presentan una respuesta absoluta. En promedio, los efectos de los cambios en la tasa de política monetaria del banco central tardan entre cuatro y seis trimestres en transmitirse completamente a la economía mediante ajustes. En las Bahamas, la reacción a estos cambios es absoluta a corto y largo plazo, debido al bajo costo de ajuste unido a la persuasión moral. 


\section{I}

\section{Introducción}

Todo gobierno puede utilizar como instrumentos mínimos la política fiscal, la política monetaria o ambas para producir un efecto en los agregados macroeconómicos del país, como la tasa de inflación, el desempleo y el crecimiento económico. Mientras que los impuestos y los gastos gubernamentales son las principales herramientas de la política fiscal, las tasas de interés y la oferta de dinero son los instrumentos clave de la política monetaria. La elección de una u otra política o de una combinación de ambas depende de las características del país, por ejemplo, el régimen cambiario, el nivel de desarrollo y el tamaño de la economía.

En este estudio se aborda el tema de la transmisión de las tasas de interés en el contexto de dos economías pequeñas con regímenes de cambio fijo: Barbados y las Bahamas. Específicamente, se analiza la dinámica de la reacción de las tasas sobre los préstamos de los bancos comerciales ante los cambios de la tasa de política monetaria de los bancos centrales de Barbados y las Bahamas, entre 1995 y 2007, con frecuencia trimestral. Por consiguiente, se estudia la eficacia de las tasas de interés para influir en la economía en ambos países. En particular, se hace hincapié en los siguientes interrogantes: ¿La tasa de política monetaria del banco central afecta a las tasas sobre los préstamos de los bancos comerciales? De ser así, ¿qué alcance tiene la transmisión de las tasas de interés a corto y largo plazo en cada economía? ¿Cuál es el tiempo mínimo de respuesta de las tasas medias sobre los préstamos de los bancos comerciales ante las acciones del banco central? ¿Cuáles son las diferencias entre ambos países en términos de política monetaria?

Como se señala en varios documentos, la vital influencia de los bancos centrales en las condiciones del mercado monetario, en especial en las tasas de interés, los ubica en el centro de la política monetaria. Los cambios en las tasas de interés del mercado monetario afectan a las tasas del mercado con períodos de madurez más largos y a las de los bancos minoristas, aunque en grado variable. Las decisiones bancarias relativas al pago de rentas sobre activos y pasivos repercuten en el gasto y las inversiones de los titulares y, en consecuencia, en la actividad económica real. En otras palabras, una transmisión más rápida y absoluta de las tasas de interés oficiales y del mercado a las de los bancos minoristas fortalece la transmisión de la política monetaria y puede incidir en la estabilidad de los precios. Asimismo, los precios que establecen los bancos influyen en sus márgenes y, por lo tanto, en su rentabilidad; por ende, afectan también a la solidez del sistema bancario y la estabilidad financiera (De Bondt, 2005, págs. 37 y 38).

El presente estudio se centra principalmente en las tasas de interés sobre los préstamos debido a que, de todas las tasas de los bancos comerciales, estas son el principal canal de transmisión de la política monetaria a la economía real, ya que constituyen una importante guía para la toma de decisiones en materia de inversión. ${ }^{1}$ Esta opinión se sustenta en los trabajos de Borio y Fritz (1995, pág. 3) y de Oliner y Rudebusch (1995, pág. 3). Por ejemplo, en este último estudio se señala que la transmisión de las tasas sobre los préstamos tiene lugar cuando las acciones del banco central afectan a la oferta de préstamos de las instituciones depositarias (los bancos) y, a su vez, al gasto real de los prestatarios.

En un gran número de trabajos se han documentado diversos grados de rigidez de las tasas de interés (Moazzami, 1999; Cottarelli y Kourelis, 1994; De Bondt, 2005; Kwapil y Scharler, 2006). La variación de la transmisión de la tasa de interés se debe, entre otros factores, a los distintos costos de ajuste, la elasticidad de la demanda de préstamos, los contratos implícitos entre bancos y clientes, los costos de cambio y los costos de información asimétrica (véase, entre otros, De Bondt, 2005; Kwapil y Scharler, 2006). En otras palabras, el grado de rigidez de las tasas sobre los préstamos depende de: i) la capacidad de los bancos comerciales de aislar totalmente su oferta de préstamos en respuesta a los cambios de las reservas, y ii) la capacidad de los prestatarios de proteger sus gastos de las alteraciones en el acceso a los préstamos bancarios (Oliner y Rudebusch, 1995, pág. 3).

Al menos tres consideraciones justifican el presente estudio. Primero, la preocupación manifestada por el Presidente del Banco Central de Barbados acerca de la

\footnotetext{
${ }^{1}$ La tasa sobre préstamos es uno de los factores determinantes de la inversión extranjera directa (IED) en Barbados, según se comprobó mediante un modelo de corrección de errores que indica que la tasa media ponderada sobre el total de los créditos causa a lo Granger a la IED en el período 1995-2005. La relación entre las dos variables es significativa (los resultados están disponibles para quien los solicite).
} 
aparente lentitud en la respuesta de las tasas sobre los préstamos de los bancos comerciales ante las recientes bajas de la tasa de interés mínima sobre los depósitos (Barbados Business Authority, 2008, pág. 1) sugiere la necesidad de una investigación cuantitativa en que se esclarezca el problema. Segundo, el entendimiento de la transmisión de la tasa de interés permite conocer mejor el mecanismo de transmisión de la política monetaria y podría brindar a los encargados de formular políticas una idea general del momento en que una determinada medida de política puede afectar a la economía real. Tercero, un estudio comparativo de países como las Bahamas y Barbados es de suma importancia, ya que puede ayudar a revelar el papel efectivo de la política monetaria en economías similares.

Para responder a los interrogantes planteados en este estudio, se emplea un modelo de corrección de errores derivado de un modelo de ajuste parcial. Este modelo, frecuentemente utilizado, se eligió con el único fin de evaluar su eficacia para capturar el fenómeno de la transmisión en Barbados. En este informe se hacen dos aportes. En primer lugar, dado que la mayoría de los estudios se enfocan en los países desarrollados, el presente estudio, al tratar el tema de las pequeñas economías del Caribe, contribuye al escaso conocimiento acerca del sector bancario de los países en desarrollo. En segundo lugar, constituye uno de los pocos estudios en que se comparan dos pequeñas economías abiertas con regímenes de tipo de cambio fijo.

El trabajo se organiza de la siguiente manera: en la sección II se hace una breve introducción a las economías de las Bahamas y Barbados, poniendo énfasis en la evolución de la tasa de interés del banco central y de los bancos comerciales. En la sección III se examina la literatura existente. La sección IV se centra en la modelización. En la sección V se presentan los resultados y las interpretaciones del ejercicio y, en la sección VI, las conclusiones finales.

\section{II}

\section{Hechos estilizados}

Las Bahamas y Barbados son islas con características económicas similares. Específicamente, son pequeñas economías abiertas cuyas monedas han estado relacionadas con el dólar de los Estados Unidos a una paridad de 2 dólares de Barbados por 1 dólar de los Estados Unidos y 1 dólar de las Bahamas por 1 dólar de los Estados Unidos. Asimismo, el crecimiento económico de ambos países está firmemente respaldado por un dinámico sector turístico - que representa aproximadamente el $60 \%$ del producto interno bruto (PIB) en las Bahamas y el $15 \%$ en Barbados-y sólidos servicios financieros.

Los bancos comerciales mantienen una posición dominante en el sistema financiero de ambos países. Debido a que representan la principal fuente de financiamiento de la economía, las tasas de interés que establecen estos bancos tienen un fuerte efecto en las decisiones sobre inversión y consumo de las personas y las empresas y, por extensión, en el desarrollo económico de estos países. Por lo tanto, la política monetaria de los bancos centrales de las Bahamas y Barbados se ha orientado, en gran medida, a influir en la evolución de las tasas de interés en el sector de la banca comercial. En las subsecciones siguientes se analizan las tendencias de los datos relativos a tasas de interés seleccionadas en las Bahamas y Barbados en el período comprendido entre 1995 y 2007.

\section{Barbados}

El Banco Central de Barbados emplea actualmente una combinación de instrumentos directos e indirectos para influir en las tasas de interés. Entre estos se incluyen una tasa de ahorro mínima, una tasa de descuento y encajes legales. La tasa de interés mínima sobre los depósitos ha constituido la principal herramienta de política monetaria desde la década de 1990, en tanto que las modificaciones en la tasa de descuento y el encaje legal cumplieron un papel secundario. Antes de 1991, el sector bancario estaba sujeto a otra serie de restricciones y reglamentaciones, como programas orientados a canalizar fondos hacia sectores prioritarios de la economía, con tasas de ahorro mínimas y topes en las tasas medias ponderadas sobre los préstamos, impuestos por el Banco Central de Barbados. En consecuencia, los cambios en las tasas de interés de los bancos comerciales por lo general los determinaba el banco central.

A comienzos de la década de 1990, la evolución macroeconómica de Barbados se caracterizó por la 
agitación de la economía mundial a causa de la guerra del Golfo y la recesión en los principales lugares de origen del turismo de Barbados: América del Norte y el Reino Unido. La economía de Barbados experimentó una marcada caída en la actividad económica real y déficits elevados persistentes en la cuenta corriente, que ocasionaron pérdidas significativas en las reservas de divisas. A fin de combatir la escasez de divisas, en mayo de 1991 el gobierno inició un programa de estabilización de 18 meses con el Fondo Monetario Internacional (FMI) que impulsó la reforma del sector financiero. Cabe resaltar que se eliminó el tope de las tasas medias de interés sobre los préstamos.

El sector financiero siguió experimentando reformas a lo largo de la década de 1990. La orden sobre las tasas de interés se revocó en junio de 1992, la tasa de interés hipotecaria residencial se desreguló en septiembre de ese año y todos los topes y controles se habían eliminado a mayo de 1993. Durante el resto de la década hubo moderadas fluctuaciones en las tasas mínimas sobre los depósitos, a medida que la política monetaria se orientaba a controlar el nivel de liquidez del sistema financiero. No obstante, las tasas de interés sobre los préstamos se mantuvieron bastante estables (con una fluctuación de alrededor del 11\%), lo que sugiere que la transmisión de los cambios en la política monetaria a estas tasas sobre los préstamos fue relativamente baja luego de su liberalización.

Entre 2000 y 2004, el sector bancario se caracterizó por los altos niveles de liquidez, consecuencia del lento crecimiento del crédito. Además, la economía cayó en recesión en 2001. Con la intención de poner freno al exceso de liquidez y estimular el préstamo destinado a sectores más productivos, el Banco Central de Barbados siguió relajando su postura de política y entre 2000 y 2001 redujo cuatro veces su tasa mínima de depósito. Sin embargo, las tasas de los bancos comerciales no se modificaron. Por consiguiente, el banco central intentó nuevamente regular las tasas sobre los préstamos y ordenó a los bancos comerciales que establecieran un tope a las tasas de interés medias ponderadas sobre los préstamos destinados a los sectores productivos. La tasa media ponderada indicativa sobre los préstamos tuvo un descenso paulatino de un máximo del $10 \%$ en agosto de 2001 a un 8,5\% en diciembre, aunque las tasas sobre los préstamos permanecieron relativamente elevadas a pesar de que el banco central siguió reduciendo su tasa mínima sobre los depósitos, la que alcanzó un punto mínimo histórico del 2,25\% en el primer trimestre de 2004. En consecuencia, la entidad volvió a introducir topes en la tasa sobre los préstamos en diciembre de
2002, estableciendo una tasa media máxima del 8,0\% en préstamos seleccionados, que rápidamente se tradujo en una caída de las tasas sobre los préstamos. Sin embargo, en 2003 esta regulación se eliminó.

Cuatro años de relativa calma en la política monetaria estimularon una fuerte demanda de crédito a lo largo de 2005 y 2006, lo que condujo a una marcada restricción de la liquidez del sistema bancario. En respuesta, el banco central elevó notoriamente la tasa mínima sobre los depósitos, del 2,25\% a comienzos de 2005 al 5,25\% a fines de 2006, lo que produjo una tendencia alcista generalizada en las tasas sobre los préstamos. A partir del cuarto trimestre de 2006 comenzó a aumentar la liquidez en el sistema bancario, reflejando en gran medida la abundante afluencia de capital extranjero y la desaceleración de la demanda de créditos. Con el objetivo de reducir la acumulación de liquidez, el banco central relajó la política monetaria rebajando la tasa mínima de los depósitos al 4,75\% a fines de 2007. En general, los bancos comerciales respondieron con lentitud a las medidas del banco central, ya que la modificación de las tasas de interés sobre los préstamos fue solo una mínima parte de los cambios acumulativos que experimentaron las tasas mínimas sobre los depósitos.

\section{Las Bahamas}

Al igual que en Barbados, la política monetaria en las Bahamas giró en torno del mantenimiento de la paridad fija entre su moneda y el dólar de los Estados Unidos, que existía desde 1973. Esta paridad fija se mantiene en la práctica conservando las reservas externas en un 50\% del valor total de billetes y monedas y obligaciones a la vista del banco central (Banco Central de las Bahamas, 1999). Desde su establecimiento, esta entidad ha aplicado controles (que incluyen topes a las tasas de interés sobre los depósitos, una tasa de descuento y disposiciones sobre tasas preferenciales) junto con la persuasión moral para influir en las tasas de interés internas. Sin embargo, los altos niveles de liquidez permitieron la eliminación del tope de las tasas en abril de 1994.

A fines de la década de 1990, el control sobre el crédito directo y la persuasión moral fueron los principales instrumentos de la política monetaria, en tanto que la política de tasas de interés pasó a cumplir un papel secundario. De hecho, la tasa de descuento solo registró dos modificaciones en el período analizado, vinculadas principalmente a los cambios de la liquidez bancaria y las condiciones del crédito interno.

La característica más destacable de la política de tasas de interés en las Bahamas es la sincronización 
entre la tasa de descuento y la tasa preferencial. Durante el período 1995-2007, la diferencia entre la tasa preferencial y la tasa del banco fue de 25 puntos básicos en cada trimestre. En principio, la tasa preferencial de los bancos comerciales está ligada a la tasa de descuento del banco central y de este vínculo surgen las posibilidades de que afecte a las tasas de interés internas y la actividad económica.

\section{III}

\section{Reseña de la bibliografía existente}

Se ha publicado un gran número de trabajos sobre la transmisión de las tasas de interés. Sin desestimar el papel de las tasas de interés sobre los depósitos de los bancos comerciales, esta reseña se centrará en las tasas sobre los préstamos. ${ }^{2}$ En términos de metodología, la mayoría de los estudios tienen como eje las series cronológicas y en ellos se utiliza un modelo de corrección de errores derivado de un modelo autorregresivo de retardos escalonados para estudiar la eficacia de los mecanismos de transmisión de la tasa de interés. Más específicamente, un estudio típico comienza de manera implícita con el modelo de Cottarelli y Kourelis (1994) o sus variantes:

$$
\begin{gathered}
L R_{t}=c+\alpha_{1} L R_{t-1}+\beta_{0} D R_{t}+\beta_{1} D R_{t-1} \\
\quad+\beta_{2} D R_{t-2}+\cdots+\beta_{n} D R_{t-n}+u_{t}
\end{gathered}
$$

donde $L R_{t}$ es la tasa de interés sobre los préstamos, $D R_{t}$ es la tasa de interés del banco central, $u_{t}$ es el término de error y $n$ representa el retardo óptimo.

La ecuación (1) es un modelo autorregresivo de retardos escalonados de orden $1(n: A D L(1, n))$. El multiplicador de impacto o de corto plazo es $\beta_{0} \mathrm{y}$ el multiplicador de largo plazo es $\beta=\sum_{i=0}^{n} \beta_{i} /\left(1-\alpha_{1}\right)$.

La ecuación (2) brinda un modelo generalizado de corrección de errores para la ecuación (1):

$$
\begin{aligned}
\Delta L R_{t}= & c+\sum_{i=1}^{n} \alpha_{i} \Delta L R_{t-i}+\sum_{i=0}^{n} \beta_{i} \Delta D R_{t-i} \\
& +\gamma\left(L R_{t-1}-\delta D R_{t-1}\right)+u_{t}
\end{aligned}
$$

\footnotetext{
2 De Bondt, Mojon y Valla (2005) demostraron que en la zona del euro las tasas sobre los depósitos, en general, no anticipaban el comportamiento de las tasas sobre los préstamos.
}

donde $\beta_{0}$ es el multiplicador de corto plazo, $\delta=\beta=\sum_{i=0}^{n} \beta_{i} /\left(1-\sum_{i=0}^{n} \alpha_{i}\right)$ es el multiplicador de largo plazo, $\gamma$ es el coeficiente de ajuste y $M=\left(1-\beta_{0}\right) / \gamma$ es el retardo de ajuste medio mediante el cual la tasa oficial se transmite a las tasas sobre los préstamos. Por medio de $\beta_{0}=1$ o de $\delta=1$ se evalúa si la transmisión es absoluta.

Los cuadros 1, 2 y 3 contienen los resultados empíricos de la transmisión a corto y a largo plazo en la zona del euro, los Estados Unidos y el Canadá y otras zonas, respectivamente. La mayoría de los autores han empleado series cronológicas con datos mensuales. Cabe destacar que las magnitudes de los multiplicadores no son necesariamente comparables, ya que los períodos y las fuentes de datos utilizados son diferentes.

En los cuadros 1, 2 y 3 se observa que, en la mayoría de los casos, la repercusión a corto plazo de la transmisión de las tasas de interés es menor que a largo plazo. En otras palabras, si bien la transmisión a corto plazo suele ser incompleta, tiende a ser más completa a largo plazo. Esto implica que la política monetaria parece ser eficaz solo a largo plazo. En apenas 3 de 44 casos los efectos son mayores a corto plazo que a largo plazo, por lo que la insostenibilidad de la transmisión de la tasa de interés es un tema discutible. La transmisión absoluta de la tasa de interés se logra a corto y a largo plazo en un número insignificante de casos; cabe afirmar que una transmisión absoluta y sostenible es, sin dudas, lo ideal.

El estudio de Sorensen y Werner (2006) es el único en que se utiliza una metodología de datos de panel para la zona del euro. Como era de esperar, se revela un alto nivel de fragmentación en el sector de la banca minorista de esa zona. Asimismo, se descubrió una elevada variación en la transmisión de la tasa de interés a nivel nacional y cierto grado de rigidez en la reacción de las tasas sobre los préstamos ante los cambios en las tasas del mercado. 
CUADRO 1

Zona del euro: transmisión de las tasas de interés, según distintos estudios

\begin{tabular}{|c|c|c|c|c|c|}
\hline Préstamos de corto plazo a empresas & Efecto & Bélgica & Francia & Alemania & Zona del euro \\
\hline Cottarelli y Kourelis (1994) & $\begin{array}{l}\text { A corto plazo } \\
\text { A largo plazo }\end{array}$ & $\begin{array}{l}0,67 \\
0,87\end{array}$ & & $\begin{array}{l}0,61 \\
0,83\end{array}$ & $\begin{array}{l}0,75 \\
0,90\end{array}$ \\
\hline Mojon (2000) & $\begin{array}{l}\text { A corto plazo } \\
\text { A largo plazo }\end{array}$ & $\begin{array}{l}1,00 \\
1,00\end{array}$ & $\begin{array}{l}0,71 \\
1,00\end{array}$ & & $\begin{array}{l}0,61 \\
1,00\end{array}$ \\
\hline Donnay y Degryse (2001) & $\begin{array}{l}\text { A corto plazo } \\
\text { A largo plazo }\end{array}$ & $\begin{array}{l}0,85 \\
0,92\end{array}$ & $\begin{array}{l}0,66 \\
0,72\end{array}$ & $\begin{array}{l}0,36 \\
0,42\end{array}$ & $\begin{array}{l}0,58 \\
0,74\end{array}$ \\
\hline Toolsema, Sturm y De Haan (2001) & $\begin{array}{l}\text { A corto plazo } \\
\text { A largo plazo }\end{array}$ & $\begin{array}{l}0,76 \\
1,02\end{array}$ & $\begin{array}{l}0,53 \\
0,62\end{array}$ & & $\begin{array}{l}0,70 \\
0,80\end{array}$ \\
\hline Heinemann y Schüller (2002) & $\begin{array}{l}\text { A corto plazo } \\
\text { A largo plazo }\end{array}$ & $\begin{array}{l}0,83 \\
1,00\end{array}$ & $\begin{array}{l}0,45 \\
1,00\end{array}$ & & $\begin{array}{l}0,75 \\
1,00\end{array}$ \\
\hline Angeloni y Ehrmann (2003) & $\begin{array}{l}\text { A corto plazo } \\
\text { A largo plazo }\end{array}$ & & & & $\begin{array}{l}0,53 \\
1,00\end{array}$ \\
\hline De Bondt (2005) & $\begin{array}{l}\text { A corto plazo } \\
\text { A largo plazo }\end{array}$ & & & & $\begin{array}{l}0,19 \\
0,88\end{array}$ \\
\hline
\end{tabular}

\begin{tabular}{|c|c|c|c|c|c|}
\hline Préstamos de largo plazo a empresas & Efecto & Bélgica & Francia & Alemania & Zona del euro \\
\hline Mojon (2000) & $\begin{array}{l}\text { A corto plazo } \\
\text { A largo plazo }\end{array}$ & $\begin{array}{l}0,61 \\
1,00\end{array}$ & $\begin{array}{l}0,42 \\
1,00\end{array}$ & & $\begin{array}{l}0,37 \\
1,00\end{array}$ \\
\hline Donnay y Degryse (2001) & $\begin{array}{l}\text { A corto plazo } \\
\text { A largo plazo }\end{array}$ & $\begin{array}{l}0,21 \\
0,10\end{array}$ & $\begin{array}{l}0,23 \\
0,50\end{array}$ & $\begin{array}{l}0,25 \\
0,60\end{array}$ & $\begin{array}{l}0,54 \\
0,67\end{array}$ \\
\hline Toolsema, Sturm y De Haan (2001) & $\begin{array}{l}\text { A corto plazo } \\
\text { A largo plazo }\end{array}$ & $\begin{array}{l}0,72 \\
0,90\end{array}$ & $\begin{array}{l}0,08 \\
0,89\end{array}$ & $\begin{array}{l}0,31 \\
0,71\end{array}$ & \\
\hline Angeloni y Ehrmann (2003) & $\begin{array}{l}\text { A corto plazo } \\
\text { A largo plazo }\end{array}$ & & & & $\begin{array}{l}0,74 \\
1,30\end{array}$ \\
\hline Kwapil y Scharler (2006) & $\begin{array}{l}\text { A corto plazo } \\
\text { A largo plazo }\end{array}$ & & & & $\begin{array}{l}0,79 \\
0,57\end{array}$ \\
\hline Kaufmann y Scharler (2006) & $\begin{array}{l}\text { A corto plazo } \\
\text { A largo plazo }\end{array}$ & & & & $\begin{array}{l}0,92 \\
1,00\end{array}$ \\
\hline De Bondt (2005) & $\begin{array}{l}\text { A corto plazo } \\
\text { A largo plazo }\end{array}$ & & & & $\begin{array}{l}0,55 \\
0,80\end{array}$ \\
\hline
\end{tabular}

Fuente: elaboración propia sobre la base de los estudios citados.

a Versión 2002.

CUADRO 2

Estados Unidos y el Canadá: transmisión de las tasas de interés, según distintos estudios

\begin{tabular}{|c|c|c|c|c|}
\hline & \multicolumn{2}{|c|}{ Estados Unidos } & \multicolumn{2}{|c|}{ Canadá } \\
\hline & $\begin{array}{l}\text { Efecto a } \\
\text { corto plazo }\end{array}$ & $\begin{array}{c}\text { Efecto a } \\
\text { largo plazo }\end{array}$ & $\begin{array}{l}\text { Efecto a } \\
\text { corto plazo }\end{array}$ & $\begin{array}{c}\text { Efecto a } \\
\text { largo plazo }\end{array}$ \\
\hline \multicolumn{5}{|c|}{ Tasas sobre préstamos a largo plazo } \\
\hline Cottarelli y Kourelis (1994) ${ }^{\mathrm{a}}$ & 0,41 & 0,97 & 0,78 & 0,93 \\
\hline Moazzami (1999) & 0,34 & 1,05 & 0,66 & 0,95 \\
\hline \multicolumn{5}{|c|}{ Tasas sobre préstamos a corto plazo } \\
\hline Moazzami (1999) ${ }^{\mathrm{c}}$ & 0,42 & 1,07 & 0,52 & 0,80 \\
\hline Kwapil y Scharler (2006) & 0,79 & 0,57 & & \\
\hline Kaufmann y Scharler (2006) & 0,92 & 1,00 & & \\
\hline
\end{tabular}

Fuente: elaboración propia sobre la base de los estudios citados.

a No existe información definida sobre la duración de los préstamos.

b Tasa de interés sobre bonos del Tesoro a tres meses.

c Tasa de interés interbancaria. 
Países seleccionados: transmisión de las tasas de interés, según distintos estudios

\begin{tabular}{|c|c|c|c|}
\hline & País & Efecto a corto plazo & Efecto a largo plazo \\
\hline Acheampong (2004) & Ghana & 0,26 & 0,55 \\
\hline Cottarelli y Kourelis (1994) & Jamaica & 0,15 & 0,92 \\
\hline Cottarelli y Kourelis (1994) & Sudáfrica & 0,61 & 1,00 \\
\hline Cottarelli y Kourelis (1994) & Venezuela (República Bolivariana de) & 0,38 & 1,48 \\
\hline
\end{tabular}

Fuente: elaboración propia sobre la base de los estudios citados.

En ciertos estudios se ha reconocido el tema de la transmisión asimétrica de la tasa de interés, es decir, la posibilidad de que las tasas sobre los préstamos respondan de modo diferente luego de un aumento o una disminución de las tasas del mercado. Sin embargo, los resultados no son concluyentes, ya que algunos autores descubrieron una asimetría en la transmisión y otros no (véanse, entre otros, Mojon, 2000; Borio y Fritz, 1995; Acheampong, 2004).
En lo que respecta a las Bahamas y Barbados, no se conoce ningún trabajo en que se haya tratado explícitamente el tema. No obstante, cabe mencionar dos estudios en que se analiza un tema relacionado. Moore y Craigwell (2002) demostraron que el poder del mercado es el principal factor determinante de la transmisión de las tasas de interés en Barbados y el Caribe. Samuel y Valderrama (2006) descubrieron también que en Barbados la política monetaria fue un factor clave en esta temática.

\section{IV}

\section{El modelo de transmisión de tasas de interés}

\section{Algunos antecedentes teóricos}

Asumamos que los bancos comerciales anticipan algunos cambios en la tasa de interés mínima del banco central, posiblemente a causa de ciertas condiciones de la economía, como las brechas entre la tasa de inflación y la producción, que afectan a la política de tasas de interés mínimas del banco central. Es muy probable que ante esa expectativa se desencadene en los bancos comerciales un cambio en las tasas de interés sobre los préstamos, en las tasas sobre los depósitos o en ambas. Debido a las razones expuestas en la introducción, en este estudio se examinarán las tasas sobre los préstamos.

Si consideramos que $L R_{t}^{*}$ es el nivel deseado de la tasa sobre los préstamos y $D R_{t}$ es la tasa mínima del banco central, la relación a largo plazo entre la tasa sobre los préstamos y la tasa mínima sobre los depósitos puede expresarse de la siguiente manera:

$$
L R_{t}^{*}=\alpha+\beta D R_{t}+e_{t}
$$

donde $t$ representa el tiempo y $\beta$ es el efecto a largo plazo de los cambios en la tasa mínima del banco central. Los efectos a largo plazo dependen de la elasticidad de la demanda de préstamos y depósitos, el grado de poder de mercado, los costos de cambio (de información, de búsqueda y administrativos) y los costos de información asimétrica (selección adversa y riesgo moral) (véase, entre otros, De Bondt, 2005, págs. 43-45). Para que la ecuación (3) funcione, debe detallarse el mecanismo de ajuste.

\section{Modelo de ajuste parcial}

En la ecuación (3), $L R_{t}^{*}-L R_{t}$ representa el cambio deseado de la tasa sobre los préstamos. Un modelo posible para este tipo de ajuste es el de ajuste parcial, que puede expresarse de la siguiente manera:

$$
L R_{t}-L R_{t-1}=\lambda\left(L R_{t}^{*}-L R_{t-1}\right)
$$

donde $0 \leq \lambda \leq 1$ es el coeficiente de ajuste, $L R_{t}-L R_{t-1}$ representa el cambio real de la tasa sobre los préstamos y $L R_{t}^{*}-L R_{t-1}$ es el cambio deseado de esta tasa.

La ecuación (4) expresa el cambio real de la tasa sobre los préstamos entre $t-1$ y $t$ como una fracción del cambio deseado en el mismo período. Nótese que si $\lambda=1$, el ajuste es instantáneo, en tanto que si $\lambda=0$, no 
hay ajuste ni cambio en la tasa sobre los préstamos, ya que $L R_{t}-L R_{t-1}$. Un costo de ajuste elevado implica un bajo coeficiente de ajuste y, a la inversa, un bajo costo de ajuste produce un coeficiente de ajuste elevado.

$\mathrm{Al}$ resolver $L R_{t}^{*}$ en la ecuación (4), se obtiene:

$$
L R_{t}^{*}=\frac{1}{\lambda} L R_{t}-\frac{(1-\lambda)}{\lambda} L R_{t-1}
$$

Al sustituir la ecuación (5) por la (3), se obtiene:

$$
L R_{t}=\alpha \lambda+\beta \lambda D R_{t}+(1-\lambda) L R_{t-1}+\lambda e_{t}
$$

donde $\beta_{0}=\beta \lambda$ es el multiplicador de corto plazo y $\beta=\frac{\beta_{0}}{\lambda}$ es el multiplicador de largo plazo. Como puede observarse, tanto los efectos a corto plazo como los de largo plazo dependen del costo de ajuste obtenido por medio del coeficiente de ajuste. La ecuación (6) es un modelo autorregresivo de retardos escalonados de orden 1,0 o simplemente un proceso autorregresivo, y también una representación reducida de un modelo de retardos escalonados infinito:

$$
L R_{t}=c+\sum_{i=0}^{\infty} \beta_{i} D R_{t-i}+e_{t}
$$

\section{Modelo de corrección de errores}

La ecuación (6) también puede expresarse restando $L R_{t-1}$ en ambos lados:

$$
\Delta L R_{t}=\alpha \lambda+\beta \lambda D R_{t}-\lambda L R_{t-1}+\lambda e_{t}
$$

donde $\Delta$ representa el operador de la primera diferencia.
La ecuación (8) puede reescribirse sumando y restando $\beta \lambda D R_{t-1}$ :

$\Delta L R_{t}=\alpha \lambda+\beta \lambda \Delta D R_{t}-\lambda\left(L R_{t-1}-\beta D R_{t-1}\right)+\lambda e_{t}$

donde $\beta_{0}=\beta \lambda$, es el multiplicador de corto plazo, $\beta$ es el multiplicador de largo plazo, $\lambda$ es el coeficiente de ajuste, $(1-\lambda) / \lambda$ es el retardo de ajuste medio y $-\log (2(1-\lambda)) / \log (1-\lambda)$ es la mediana del retardo.

La ecuación (9) es el modelo de corrección de errores correspondiente al modelo autorregresivo de retardos escalonados de orden $(1,0)$ capturado en la ecuación (6). Como puede observarse, no se presenta la teoría de cointegración para la ecuación (9), ya que el modelo de corrección de errores es anterior a esta. Básicamente, si las variables son fijas en nivel, tanto la ecuación (6) como la (9) son representaciones válidas del fenómeno estudiado.

\section{Método de estimación}

Como ya se señaló, las ecuaciones (6) y (9) son equivalentes y tienen parámetros altamente no lineales. Por lo tanto, se requieren algunos algoritmos no lineales para estimarlas. Además, se debe prestar especial atención al tema de la autocorrelación y la heterocedasticidad.

Cabe recordar que el objetivo de la estimación del modelo es obtener la transmisión a corto plazo de la tasa de interés: $\beta_{0}=\beta \lambda$, la transmisión a largo plazo: $\beta$, y el retardo medio: $(1-\lambda) / \lambda$. Asimismo, debido a las razones explicadas en Hendry (1995, págs. 216 y 257), también se deriva una mediana del retraso.

\section{V}

\section{Resultados empíricos}

Los datos de interés son los siguientes: para Barbados, la tasa mínima de los depósitos del Banco Central de Barbados y la tasa media ponderada sobre el total de los préstamos y la tasa media ponderada sobre préstamos seleccionados; para las Bahamas, la tasa del Banco Central de las Bahamas y la tasa de interés media ponderada sobre préstamos y líneas de crédito a los clientes de los bancos comerciales.

Comenzaremos con Barbados, examinando las propiedades de las series cronológicas de estas variables en el período comprendido entre 1995 y 2007, con frecuencia trimestral. En el gráfico 1 se observa la evolución de cada serie. La media trimestral se ubica en un $4,029 \%$, un $11,55 \%$ y un $9,87 \%$, respectivamente, para la tasa mínima de los depósitos del Banco Central de Barbados, la tasa media ponderada sobre el total de los préstamos y la tasa media ponderada sobre préstamos seleccionados; en tanto que la mediana es de un $4 \%$, $11,30 \%$ y $10,19 \%$, respectivamente. Se observa un alto grado de sincronización entre las series, reflejado por 
GRÁFICO 1

Barbados: evolución de las tasas de interés (datos trimestrales), enero de 1995-abril de 2007

(En porcentajes)

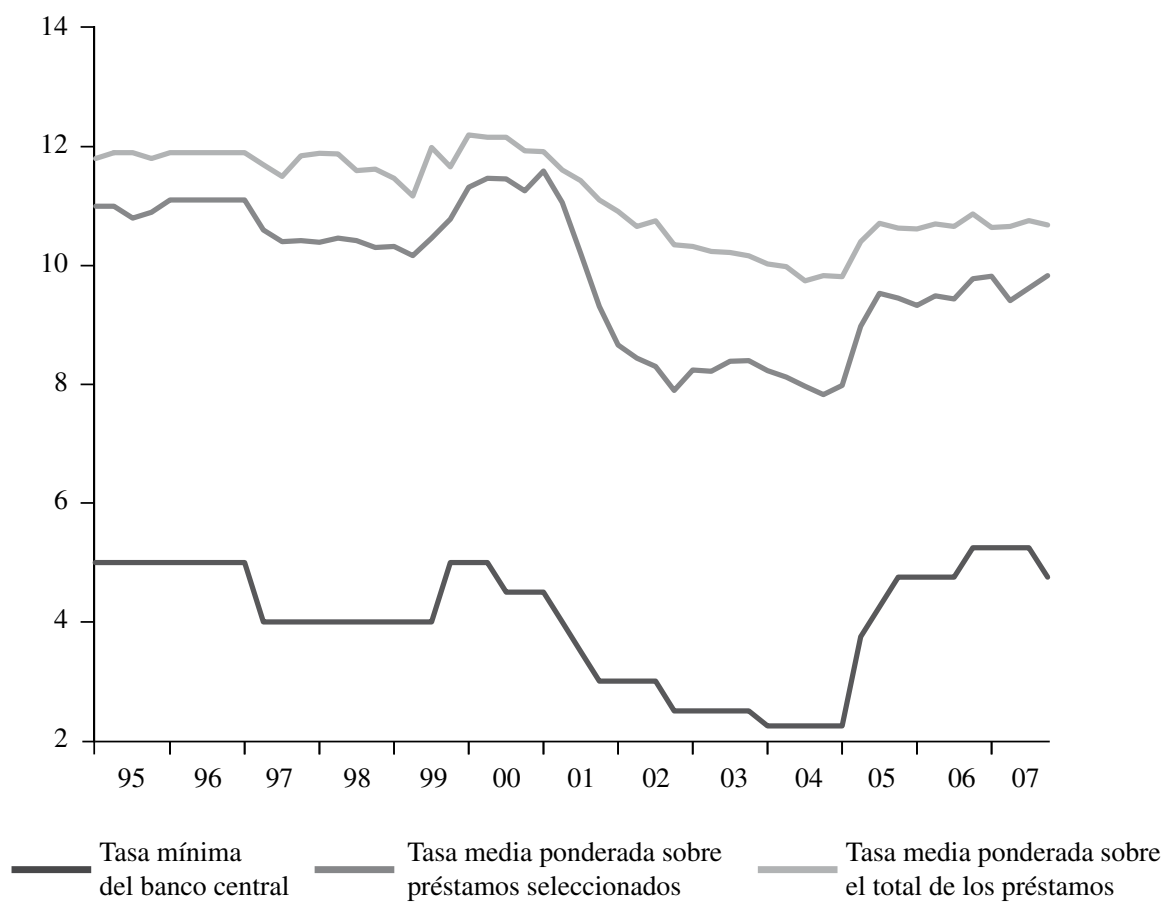

Fuente: Banco Central de Barbados, Economic and Financial Statistics.

la marcada correlación existente entre ellas: de 0,659 entre la tasa mínima oficial sobre los depósitos y la tasa media ponderada sobre el total de los préstamos; de 0,791 entre la tasa mínima oficial sobre los depósitos y la tasa media ponderada sobre préstamos seleccionados, y de 0,945 entre la tasa media ponderada sobre el total de los préstamos y la tasa media ponderada sobre préstamos seleccionados. A fin de estudiar la estacionalidad y no estacionalidad de las variables, se recurrió a dos pruebas de raíz unitaria formales: la prueba de Dickey-Fuller aumentada y la prueba de Phillips-Perron. El objetivo principal de estas es descartar cualquier regresión espuria. ${ }^{3}$ Debido a que ambas pruebas son de uso común, no se explican en este estudio.

En el cuadro 4 se advierte que las tres variables de interés son no estacionarias a un nivel de significación del 5\%. Dado que las pruebas de Dickey-Fuller aumentada

\footnotetext{
${ }^{3}$ Esta estrategia de pruebas previas fue en gran medida abandonada en el esquema elaborado recientemente por Pesaran, Shin y Smith (2001).
}

y de Phillips-Perron son mayores que el valor crítico correspondiente a cada variable en términos de nivel y menores que el valor crítico correspondiente a cada variable en la primera diferencia se puede concluir que se trata de series integradas de orden 1.

Dado que las variables son no estacionarias, surge el problema de la cointegración. En todo caso, debido a que la estimación realizada con el modelo autorregresivo de retardos escalonados en la ecuación (6), empleando la tasa mínima sobre los depósitos del banco central y la tasa media ponderada sobre el total de los préstamos como variables, conduce a los mismos resultados que el modelo de corrección de errores de la ecuación (9), solo se presentan los resultados de este último. En el ejercicio se empleó un método de estimación de mínimos cuadrados no lineales, aplicando los errores estándares robustos de Newey-West para solucionar los problemas de autocorrelación y heterocedasticidad (véanse más detalles sobre este método en el programa Eviews). En el cuadro 5 se muestran los resultados del modelo de corrección de errores para la tasa media ponderada sobre el total de los préstamos (véase la ecuación (9)). 
CUADRO 4

Barbados: resultados de las pruebas de Dickey-Fuller aumentada y de Phillips-Perron, enero de 1995-abril de 2007

(Según datos trimestrales)

\begin{tabular}{lccc}
\hline Variable & $\begin{array}{c}\text { Prueba t de } \\
\text { Dickey-Fuller } \\
\text { aumentada (nivel) }\end{array}$ & $\begin{array}{c}\text { Prueba t de } \\
\text { Phillips-Perron } \\
\text { (nivel) }\end{array}$ & $\begin{array}{c}\text { Prueba t de Dickey-Fuller } \\
\text { aumentada } \\
\text { (primera diferencia) }\end{array}$ \\
\hline $\begin{array}{l}\text { Tasa mínima sobre los } \\
\text { depósitos del banco central } \\
\text { Tasa media ponderada sobre el }\end{array}$ & $-1,747$ (c) & $-1,631$ (c) & $-5,382(0) *$ \\
$\begin{array}{l}\text { Phillips-Perron } \\
\text { Tasa de los préstamos } \\
\text { préstamos seleccionados }\end{array}$ & $-1,182$ (c) & $-1,262$ (c) & $-3,909(0) *$ \\
\hline
\end{tabular}

Fuente: cálculos obtenidos sobre la base de datos del Banco Central de Barbados, Economic and Financial Statistics, varios números.

Nota: la hipótesis nula de las pruebas de Dickey-Fuller aumentada y de Phillips-Perron es que los datos tienen una raíz unitaria; (c): con una constante solo en las ecuaciones de las pruebas de Dickey-Fuller aumentada y de Phillips-Perron en nivel; los valores críticos para las pruebas de Dickey-Fuller aumentada y de Phillips-Perron con constante son (c): $-3,568,-2,921$ y $-2,599$, al 1\%, 5\% y $10 \%$ de significación, respectivamente; (0): sin constante ni tendencia temporal en las ecuaciones de primera diferencia; los valores críticos son: $-2,612,-1,948 \mathrm{y}-1,613$, al $1 \%, 5 \%$ y $10 \%$ de significación, respectivamente.

* Significación al $5 \%$.

CUADRO 5

Barbados: resultados de la corrección de errores para el promedio del total de los préstamos (datos trimestrales), enero de 1995-abril de 2007

Variable dependiente: $\Delta$ watloan

Método: cuadrados mínimos no lineales

Errores estándares de heterocedasticidad y autocorrelación de Newey-West y covarianza (truncamiento de los retardos=3)

\begin{tabular}{|c|c|c|c|c|}
\hline Parámetro & Coeficiente & Error estándar & Estadístico t & Probabilidad \\
\hline$\alpha$ & 7,950626 & 0,699985 & 11,35829 & 0,0000 \\
\hline$\lambda$ & 0,136713 & 0,055639 & 2,457132 & 0,0176 \\
\hline$\beta$ & 0,758633 & 0,150735 & 5,032896 & 0,0000 \\
\hline$R^{2}$ & 0,164425 & & & \\
\hline$D W$ & 2,43497 & & & \\
\hline $\begin{array}{l}\text { Prueba del multiplicador de Lagrange } \\
\text { de correlación serial }\left(\chi_{4}^{2}\right)^{\mathrm{a}}\end{array}$ & $\begin{array}{c}6,7604 \\
(0,1491)\end{array}$ & $\begin{array}{l}\text { Prueba de normalidad } \\
\text { de Jarque-Bera }\end{array}$ & $\begin{array}{l}62,221 \\
(0,0000)\end{array}$ & \\
\hline Prueba de heterocedasticidad de White $\left(\chi_{5}^{2}\right)$ & $\begin{array}{l}9,2490 \\
(0,0995)\end{array}$ & & & \\
\hline
\end{tabular}

Fuente: resultados econométricos sobre la base de datos del Banco Central de Barbados, Economic and Financial Statistics, varios números. Nota: el modelo es $\Delta_{t}$ watloan $=\alpha \lambda+\beta \lambda \Delta$ mdrate $_{t}-\lambda\left(\right.$ watloan $_{t-1}-\beta$ mdrate $\left._{t-1}\right)+\lambda e_{t}$, donde $\Delta$ representa al operador de la primera diferencia, watloan es la tasa media ponderada sobre el total de los préstamos, mdrate es la tasa mínima sobre los depósitos del banco central, $\lambda$ es el coeficiente de ajuste, $\beta$ es el efecto a largo plazo y $\beta \lambda$ el efecto a corto plazo. No se omitió ninguna observación.

a Cifras entre paréntesis corresponden a valores $p$.

Antes de proceder a la interpretación de los resultados principales, cabe destacar que si bien la ecuación pasa la prueba de autocorrelación, como indica el valor $p$ de la prueba del multiplicador de Lagrange, en este caso no existe heterocedasticidad ni normalidad. Sin embargo, por medio del uso de errores estándares robustos se tiene en cuenta la heterocedasticidad detectada a un nivel del 10\%. En el cuadro 5 se indica que el efecto implícito a corto plazo (elasticidad) es del 0,104\%. Con un valor $p$ de 0,0077 y un estadístico de Wald de 7,105, la elasticidad a corto plazo es estadísticamente diferente a cero al 5\% de significación. Asimismo, la elasticidad de corto plazo es también estadísticamente diferente a 1 , con un valor $p$ de 0,000 y un estadístico de Wald de 530,575. Básicamente, no parece existir una transmisión absoluta de tasas de interés a corto plazo. El efecto o la 
elasticidad de largo plazo es del 0,759\%. Este último valor es estadísticamente diferente a cero. Sin embargo, con un valor $p$ de 0,109 y un estadístico de Wald de 2,564, la elasticidad de largo plazo no es estadísticamente diferente a 1 . Los dos resultados combinados indican que si bien el impacto de transmisión a corto plazo parece no existir, a largo plazo es totalmente efectivo. El asunto clave es el grado de retardo de la transmisión. Para establecerlo, se calculó el retardo medio y la mediana del retardo. El retardo medio es de 6,314; es decir que, en promedio, el efecto de los cambios en la tasa de interés mínima demora seis trimestres en transmitirse a las tasas sobre los préstamos, en tanto que el $50 \%$ del efecto se transmite en menos de cuatro trimestres $(3,715)$.

Para comprobar si los resultados son sólidos con respecto a otros tipos de préstamos, en el cuadro 6 se presentan los resultados obtenidos con las tasas sobre préstamos seleccionados.

En el cuadro 6 puede observarse que, si bien la normalidad se satisface, no ocurre lo mismo con la heterocedasticidad y la autocorrelación. Mediante el uso de errores estándares consistentes con la heterocedasticidad y la autocorrelación de Newey-West se corrigen ambos problemas. Los resultados sugieren que el efecto implícito a corto plazo (elasticidad) es del $0,247 \%$. Con un valor $p$ de 0,0081 y un estadístico de Wald de 7,013, la elasticidad de corto plazo es estadísticamente diferente a cero. Asimismo, el efecto de corto plazo es también estadísticamente diferente a 1 , como lo indica el valor $p$ de 0,000 y un estadístico de Wald de 65,293. Específicamente, es menor a 1 , es decir que a corto plazo no existe una transmisión absoluta de tasas de interés. En el cuadro 6 también resalta que la elasticidad de largo plazo implícita es del $1,226 \%$. Este último valor es estadísticamente diferente a cero. Con un valor $p$ de 0,324 y un estadístico de Wald de 0,973 , la elasticidad a largo plazo no es estadísticamente diferente a 1 a un nivel de significación del 5\%. En resumen, la combinación de ambos resultados indica que la transmisión de la tasa de interés solo es completamente efectiva a largo plazo. El retardo medio, con un valor de 3,965 , indica que en promedio se necesitan cuatro trimestres para que el efecto de un cambio en la tasa mínima de los depósitos se transmita a las tasas de interés sobre préstamos seleccionados y que el 50\% del efecto se transmite en menos de dos trimestres.

En cuanto a las Bahamas, en el gráfico 2 se muestra que la tasa del banco central y la tasa sobre los préstamos, que es la tasa de interés media ponderada sobre los préstamos y sobregiros, disminuyen a lo largo del tiempo. La media trimestral es del 5,88\% y del 11,60\% para la tasa del banco central y la tasa de interés media ponderada sobre préstamos y descubiertos, respectivamente, en tanto que la mediana es del 5,75\% para la primera y del $11,76 \%$ para la segunda. Al igual que en Barbados, se observa un alto grado de correlación entre ambas variables $(0,85)$.

CUADRO 6

Barbados: resultados de la corrección de errores para el promedio de préstamos seleccionados (datos trimestrales), enero de 1995-abril de 2007

Variable dependiente: $\Delta$ wasloan

Método: cuadrados mínimos no lineales

Errores estándares de heterocedasticidad y autocorrelación de Newey-West y covarianza (truncamiento de los retardos=3)

\begin{tabular}{ccccc}
\hline Parámetro & Coeficiente & Error estándar & Estadístico t & Probabilidad \\
\hline$\alpha$ & 4,837868 & 0,981456 & 4,929277 & 0,0000 \\
$\lambda$ & 0,201407 & 0,075725 & 2,659700 & 0,0105 \\
$\beta$ & 1,225545 & 0,228672 & 5,359395 & 0,0000 \\
$R^{2}$ & 0,3184 & & & \\
$D W$ & 1,0308 & & & \\
Prueba del multiplicador de Lagrange & 13,2112 & Prueba de normalidad & 0,6506 \\
de correlación serial $\left(\chi_{4}^{2}\right)^{\mathrm{a}}$ & $(0,0103)$ & de Jarque-Bera & $(0,7223)$ \\
Prueba de heterocedasticidad de White $\left(\chi_{5}^{2}\right)$ & 9,7826 & & & \\
& $(0,0816)$ & & & \\
\hline
\end{tabular}

Fuente: resultados econométricos sobre la base de datos del Banco Central de Barbados, Economic and Financial Statistics, varios números. Nota: el modelo es $\Delta$ wasloan $_{t}=\alpha \lambda+\beta \lambda \Delta$ mdrate $_{t}-\lambda\left(\right.$ wasloan $_{t-1}-\beta$ mdrate $\left._{t-1}\right)+\lambda e_{t}$, donde $\Delta$ representa el operador de la primera diferencia, wasloan es la tasa media ponderada sobre préstamos seleccionados, mdrate es la tasa mínima sobre los depósitos del banco central, $\lambda$ es el coeficiente de ajuste, $\beta$ es el efecto a largo plazo y $\beta \lambda$ el efecto a corto plazo. No se omitió ninguna observación.

a Cifras entre paréntesis corresponden a valores $p$. 
GRÁFICO 2

Bahamas: evolución de las tasas de interés (datos trimestrales), enero de 1995-abril de 2007

(En porcentajes)

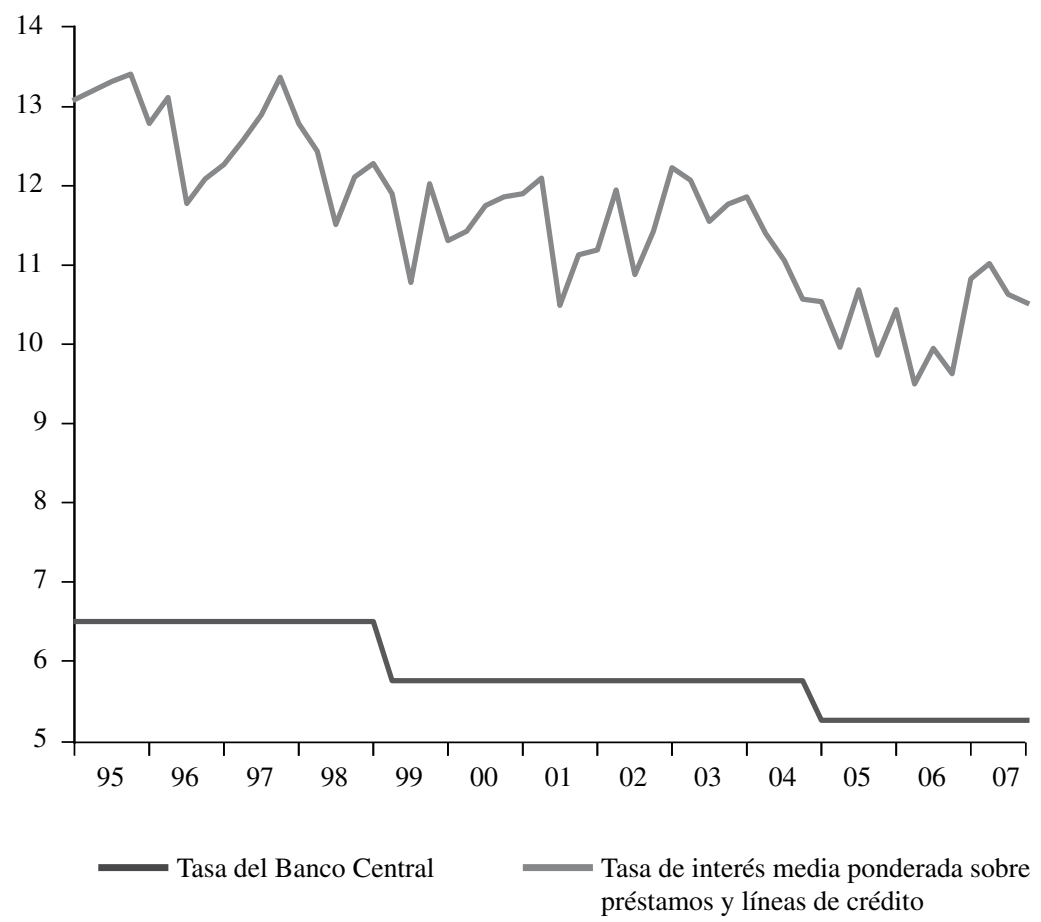

Fuente: elaboración propia sobre la base de datos del Banco Central de las Bahamas.

CUADRO 7

Bahamas: resultados de las pruebas de Dickey-Fuller aumentada y de Phillips-Perron (datos trimestrales), enero de 1995-abril de 2007

\begin{tabular}{lcccc}
\hline Variable & $\begin{array}{c}\text { Prueba t de Dickey-Fuller } \\
\text { aumentada } \\
(\text { nivel) }\end{array}$ & $\begin{array}{c}\text { Prueba t de } \\
\text { Phillips-Perron } \\
\text { (nivel) }\end{array}$ & $\begin{array}{c}\text { Prueba de Dickey-Fuller } \\
\text { aumentada } \\
\text { (primera diferencia) }^{\mathrm{a}}\end{array}$ & $\begin{array}{c}\text { Prueba de } \\
\text { Phillips-Perron } \\
\text { (primera diferencia) }\end{array}$ \\
\hline $\begin{array}{l}\text { Tasa del Banco Central de } \\
\text { las Bahamas }\end{array}$ & $-2,576^{\mathrm{b}}$ & $-2,675^{\mathrm{b}}$ & $-7,211^{\mathrm{c}, \mathrm{d}}$ & $-7,352^{\mathrm{c}, \mathrm{d}}$ \\
$\begin{array}{l}\text { Tasa de interés media } \\
\text { ponderada sobre préstamos } \\
\text { y líneas de crédito }\end{array}$ & $-4,610^{\mathrm{b}, \mathrm{d}}$ & $-4,658^{\mathrm{b}}$ & $-10,656^{\mathrm{c}, \mathrm{d}}$ & $-18,972^{\mathrm{c}, \mathrm{d}}$ \\
\hline
\end{tabular}

Fuente: elaboración propia sobre la base de datos del Banco Central de las Bahamas.

a La hipótesis nula de las pruebas de Dickey-Fuller aumentada y de Phillips-Perron es que los datos tienen una raíz unitaria.

b Con una constante y una tendencia temporal. Los valores críticos para ambas pruebas son: $-4,148,-3,500$ y $-3,174$, al $1 \%, 5 \%$ y $10 \%$ de significación, respectivamente.

c Con una constante. Los valores críticos son: $-3,568,-2,921$ y $-2,599$ al 1\%, 5\% y $10 \%$ de significación, respectivamente.

d Nivel de significación del $5 \%$.

A continuación, se examina la estacionalidad o no estacionalidad de cada variable empleando la prueba de
Dickey-Fuller aumentada y la prueba de Phillips-Perron. El cuadro 7 contiene los resultados del ejercicio. 
Según los valores de las pruebas, en las Bahamas, la tasa del banco central es no estacionaria y la tasa de interés media ponderada sobre préstamos y líneas de crédito es estacionaria. No obstante, los datos del gráfico 2 despiertan algunas dudas sobre la estacionalidad de la última variable. Tanto la prueba de KwiatkowskiPhillips-Schmidt-Shin como la de Elliot, Rothenberg y Stock, que no se presenta en este estudio, indican que la tasa de interés media ponderada sobre préstamos y líneas de crédito es no estacionaria. Aceptamos este resultado.

Cabe entonces examinar la relación entre las dos variables utilizando el modelo de corrección de errores (véase la ecuación (9). En el cuadro 8 se presentan los resultados del ejercicio.

Puede observarse que la ecuación pasa las pruebas de autocorrelación, heterocedasticidad y normalidad. El efecto o la elasticidad de corto plazo implícita que se deriva del cuadro 8 es del 1,193\%. Con un valor $p$ de 0,0000 y un estadístico de Wald de 18,881, la elasticidad de corto plazo es estadísticamente diferente a cero; sin embargo, no es estadísticamente diferente a 1 , como lo indican el valor $p$ de 0,481 y un estadístico de Wald de 0,496 al 5\% del nivel de significación, como mínimo. Es decir, a corto plazo hay un efecto de transmisión absoluta de tasas de interés. En el cuadro 8 también se muestra que la elasticidad de largo plazo implícita es del 1,780\%. Este último valor es estadísticamente diferente a cero. Con un valor $p$ de 0,0009 y un estadístico de Wald de
11,025, la elasticidad de largo plazo es estadísticamente mayor que 1 a un nivel de significación del 5\%. En resumen, la combinación de ambos resultados indica que la transmisión de la tasa de interés es completamente efectiva a corto plazo y a largo plazo. El retardo medio, con un valor de 0,507 , indica que en promedio se necesita solo medio trimestre para que el efecto de un cambio en la tasa del banco central se transmita a la tasa de interés sobre los préstamos. Esto puede deberse al alto grado de sincronización entre la tasa de política monetaria y la tasa sobre los préstamos.

En términos generales, hay tres hallazgos principales. Primero, la transmisión de la tasa de interés es absoluta a largo plazo en ambos países. Segundo, mientras que en Barbados las acciones del banco central demoran entre cuatro y seis trimestres en transmitirse a los bancos comerciales, en las Bahamas la transmisión es casi instantánea (medio trimestre). Tercero, si el Banco Central de Barbados desea una transmisión instantánea, es decir, que la tasa sobre los préstamos aumente (o se reduzca) 100 puntos básicos durante el trimestre en el que se modifica la tasa mínima sobre los depósitos, esta debe incrementarse (o reducirse) en un rango de entre 500 y 730 puntos básicos, aproximadamente. Asimismo, deben investigarse a fondo las razones de un elevado costo de ajuste. Aunque esa investigación está fuera del ámbito de este informe, se pueden citar como determinantes potenciales el poder de mercado, los costos de cambio,

CUADRO 8

Bahamas: resultados de la corrección de errores de la ecuación (9) para los préstamos (datos trimestrales), enero de 1995-abril de 2007

Variable dependiente: $\Delta$ waverate

Método: cuadrados mínimos no lineales

Errores estándares de heterocedasticidad y autocorrelación de Newey-West y covarianza (truncamiento de los retardos=3)

\begin{tabular}{|c|c|c|c|c|}
\hline Parámetro & Coeficiente & Error estándar & Estadístico t & Probabilidad \\
\hline$\alpha$ & 0,993616 & 1,398494 & 0,710490 & 0,4808 \\
\hline$\lambda$ & 0,663163 & 0,114208 & 5,806640 & 0,0000 \\
\hline$\beta$ & 1,799594 & 0,240816 & 7,472908 & 0,0000 \\
\hline$R^{2}$ & 0,3333 & & & \\
\hline$D W$ & 2,1254 & & & \\
\hline $\begin{array}{l}\text { Prueba del multiplicador de Lagrange } \\
\text { de correlación serial }\left(\chi_{4}^{2}\right)^{\mathrm{a}}\end{array}$ & $\begin{array}{c}6,0062 \\
(0,1987)\end{array}$ & $\begin{array}{c}\text { Prueba de normalidad } \\
\text { de Jarque-Bera }\end{array}$ & $\begin{array}{l}2,7015 \\
(0,2590)\end{array}$ & \\
\hline Prueba de heterocedasticidad de White $\left(\chi_{5}^{2}\right)$ & $\begin{array}{l}1,655 \\
(0,8945)\end{array}$ & & & \\
\hline
\end{tabular}

Fuente: resultados econométricos obtenidos sobre la base de datos del Banco Central de las Bahamas.

Nota: El modelo es $\Delta$ waverate $_{t}=\alpha \lambda+\beta \lambda \Delta$ brate $_{t}-\lambda\left(\right.$ waverate $_{t-1}-\beta$ brate $\left._{t-1}\right)+\lambda e_{t}$, donde $\Delta$ representa el operador de la primera diferencia, waverate es la tasa de interés media ponderada sobre préstamos y líneas de crédito, brate es la tasa del banco central, $\lambda$ es el coeficiente de ajuste, $\beta$ es el efecto a largo plazo y $\beta \lambda$ el efecto a corto plazo. No se omitió ninguna observación.

a Cifras entre paréntesis corresponden a valores $p$. 
la elasticidad de la demanda de préstamos y los costos de información asimétrica (Moore y Craigwell, 2002).

Cabe analizar dos cuestiones que podrían tener cierta repercusión en los resultados presentados: las variables omitidas y la asimetría de las respuestas de los bancos comerciales a las turbulencias monetarias positivas y negativas. Dado que las tasas sobre los préstamos se ven en teoría influidas por muchos factores además de la tasa mínima sobre los depósitos (como el poder de mercado, los costos de cambio, la elasticidad de la demanda de préstamos y los costos de información asimétrica), es importante saber si el modelo contiene errores graves de especificación de variables omitidas. ${ }^{4}$ Pueden plantearse tres consideraciones, entre otras. Primero, el modelo estimado (6 o 9) es una variante reducida de un modelo más general de retardos escalonados infinitos (7), que captura implícitamente las variables omitidas mediante las variables retardadas de la tasa mínima de los depósitos,

${ }^{4}$ Como método de aproximación a determinada realidad, un modelo siempre tendrá cierto grado de error de especificación. La cuestión clave es la magnitud de ese error.

\section{VI}

\section{Conclusiones}

Empleando un modelo de corrección de errores derivado de un modelo de ajuste parcial, en este estudio se investiga de manera empírica la eficacia de la política de tasas de interés de los bancos centrales de las Bahamas y Barbados para influir en el comportamiento de los bancos comerciales, en el período comprendido entre enero de 1995 y abril de 2007. Una respuesta no absoluta de los bancos comerciales a los cambios en la política del banco central en materia de tasas de interés impide el buen funcionamiento del sistema financiero. Mediante este estudio se descubrió que en Barbados la reacción de las tasas sobre los préstamos de los bancos comerciales a los cambios de la tasa de interés mínima del banco central es inflexible a corto plazo, pero absoluta o efectiva a largo plazo. En promedio, los efectos de los cambios en la tasa de política monetaria del banco central demoran entre cuatro y seis trimestres en transmitirse por completo a la economía por medio de ajustes.

Dada la magnitud del retardo de ajuste medio en Barbados, es prioritario encontrar el modo de lograr que ya que estas responden a la situación de la economía a lo largo de los trimestres. Segundo, el efecto de la mayoría de las llamadas variables omitidas es capturado por el coeficiente de ajuste, que está vinculado al costo de ajuste. Es decir, el coeficiente de ajuste es un tipo de estadístico de resumen de todos los tipos de variables omitidas. Por lo tanto, puede inferirse que muy probablemente el modelo no presenta un error grave de especificación de variables omitidas. Tercero, la prueba formal de especificación de errores de ecuaciones de regresión de Ramsey para la regresión lineal parece confirmar que no existen variables omitidas. Esto es válido tanto para Barbados, cuyo valor de la prueba de Ramsey es de 3,180 con un valor $p$ de 0,365 , al menos para la tasa media ponderada sobre el total de los préstamos, como para las Bahamas, con un valor de 4,369 y un valor $p$ de 0,224 .

La asimetría de las respuestas a las turbulencias monetarias positivas y negativas es un tema que reviste importancia. Si bien teóricamente un modelo asimétrico es un constructo válido, en la práctica no lo es necesariamente, como se señala en la reseña de la bibliografía. Esta línea de investigación no está incluida en el presente estudio.

la tasa sobre los préstamos sea plenamente efectiva a corto plazo. De acuerdo con los resultados del estudio, para incrementar (o reducir) las tasas sobre los préstamos en 100 puntos básicos, debe aumentarse (o disminuir) la tasa mínima de los depósitos en un rango de entre 500 y 730 puntos básicos, aproximadamente. Asimismo, deben investigarse a fondo las razones del elevado costo de ajuste. El poder de mercado, la elasticidad de la demanda de préstamos, los costos de cambio y la asimetría de los costos de información, entre otros, serían los elementos a tener en cuenta cuando se trata de acrecentar la eficacia de la política de tasas de interés.

Los métodos y la frecuencia con que el banco central comunica al público sus intenciones de política también pueden ser factores determinantes de la eficacia de la política monetaria. En otros estudios (Blinder y otros, 2008) se ha demostrado que la información de los bancos centrales sobre sus medidas es realmente relevante cuando se trata de reducir la transmisión de las turbulencias monetarias. Considerando que una comunicación 
más fluida ayuda a definir las expectativas públicas, la comunicación frecuente de un banco central sobre la orientación futura de su política monetaria permitirá asegurar una mayor claridad de las medidas a tomar.

En el caso de las Bahamas, en el estudio se descubre que la reacción de las tasas sobre los préstamos de los bancos comerciales a los cambios en la tasa de interés de política monetaria del banco central es absoluta o efectiva tanto a corto como a largo plazo. La presencia de un menor costo de ajuste, debido a la alta velocidad de ajuste combinada con la persuasión moral, puede ser en gran parte la causa de que las reacciones de la tasa de interés sean absolutas a corto y largo plazo en este país.

En líneas generales, con este estudio comparativo se demuestra que las características de la política monetaria aplicada, junto con otros factores, son el motivo de que economías similares respondan de modo diferente a las turbulencias de la política monetaria. En realidad, si bien los controles sobre la tasa de interés y la persuasión moral dieron como resultado la transmisión absoluta a corto plazo de las tasas de interés en las Bahamas, esto no ocurre en el caso de la tasa mínima sobre los depósitos de Barbados.
Acheampong, Kwasi (2004), "Bank interest rate channel of monetary policy transmission in Ghana", Bank of Ghana Working Paper, $\mathrm{N}^{\circ} 10$, agosto.

Angeloni, Ignazio y Michael Ehrmann (2003), "Monetary transmission in the euro area: early evidence", Economic Policy, vol. 18, N ${ }^{\circ} 37$, Londres, Centre for Economic Policy Research (CEPR), octubre.

Banco Central de las Bahamas (1999), The Central Bank of the Bahamas: Celebrating 25 Years of Service, 1974-1999, Nassau.

Barbados Business Authority (2008), Bridgetown, 5 de mayo.

Blinder, Alan y otros (2008), "Central Bank communication and monetary policy: a survey of theory and evidence", Working Paper Series, $\mathrm{N}^{\circ}$ 898, Frankfurt, Banco Central Europeo, mayo.

Borio, Claudio y Wilhelm Fritz (1995), "The response of shortterm bank lending rates to policy rates: a cross-country perspective", BIS Working Papers, $\mathrm{N}^{\circ} 27$, Basilea, Banco de Pagos Internacionales, mayo.

Cottarelli, Carlo y Angeliki Kourelis (1994), "Financial structure, bank lending rates and the transmission mechanism of monetary policy", IMF Staff Papers, vol. 41, N 4, Washington, D.C., Fondo Monetario Internacional.

De Bondt, Gabe, (2005), "Interest rate pass-through: empirical results for the euro area", German Economic Review, vol. 6, $\mathrm{N}^{\circ} 1$, Oxford, Blackwell Publishing, febrero.

De Bondt, Gabe, Benoit Mojon y Natacha Valla (2005), “Term structure and the sluggishness of retail bank interest rates in euro area countries", European Working Paper Series, № 518, Frankfurt, Banco Central Europeo, septiembre.

Donnay, Marie y Hans Degryse (2001), "Bank lending rate pass-through and differences in the transmission of a single EMU monetary policy", Center for Economic Studies Discussion Papers, $\mathrm{N}^{\circ} 0117$, Leuven, Katholieke Universiteit, agosto.

Heinemann, Friedrich y Martin Schüller (2002), "Integration benefits on EU retail markets: evidence from interest rate pass-through", ZEW Discussion Paper, $\mathrm{N}^{\circ}$ 02-26, Mannheim, Zentrum für Europäische Wirtschaftsforschung, abril.

Hendry, David (1995), Dynamic Econometrics, Oxford, Oxford University Press.
Kaufmann, Sylvia y Johann Scharler (2006), "Financial systems and the cost of channel transmission of monetary policy shocks", Working Papers, $\mathrm{N}^{\circ} 116$, Viena, Oesterreichische Nationalbank, marzo.

Kwapil, Claudia y Johann Scharler (2006), "Limited pass-through from policy to retail interest rates: empirical evidence and macroeconomic implications", Monetary Policy and the Economy, Q4, Viena, Oesterreichische Nationalbank.

Moazzami, Bakhtiar (1999), "Lending rate stickiness and monetary transmission mechanism: the case of Canada and the United States", Applied Financial Economics, vol. 9, N 6, Londres, Taylor and Francis, diciembre.

Mojon, Benoit (2000), "Financial structure and the interest rate channel of ЕСв monetary policy", Working Paper Series, $\mathrm{N}^{\circ} 40$, Frankfurt, Banco Central Europeo, noviembre.

Moore, Winston y Roland Craigwell (2002), "Market power and interest rate spreads in the Caribbean", International Review of Applied Economics, vol. 16, $\mathrm{N}^{\circ} 4$, Londres, Taylor and Francis.

Oliner, Stephen y Glen Rudebusch (1995), "Is there a bank lending channel for monetary policy?", Economic Review, San Francisco, Federal Reserve Bank of San Francisco.

Pesaran, M., Y. Shin y R. Smith (2001), "Bounds testing approaches to the analysis of the level relationships", Journal of Applied Econometrics, vol. 16, $\mathrm{N}^{\circ} 3$, John Wiley \& Sons.

Samuel, Wendell y Laura Valderrama (2006), "The monetary policy regime and banking spreads in Barbados", IMF Working Papers, $N^{\circ}$ 06/211, Washington, D.C., Fondo Monetario Internacional.

Sorensen, Kristopher y Thomas Werner (2006), "Bank interest rate pass-through in the euro area: a cross country comparison", Working Paper Series, N $^{\circ}$ 580, Frankfurt, Banco Central Europeo, enero.

Toolsema, Linden, Jan-Egbert Sturm y Jakob de Haan (2001), "Convergence of monetary transmission in EMU-new evidence", CESifo Working Paper Series, $\mathrm{N}^{\circ} 465$, Munich, CEsifo Group Munich, abril. 